\title{
Design and Enhancement of License Plate Images Based on Kernel Estimation using Adaptive Filter
}

\author{
B.Harichandana, K.Lavanya, P.Sumalatha, C.Krishnapriya
}

\begin{abstract}
The speed of vehicles is uncovered because of hit and run accidents are occurred. Generally, the fast moving vehicle image is captured by the surveillance camera. The images that are observed by this camera consist of low resolution and the image will be in the blur format. Because of this the information will be loss. In this paper, to overcome this issue with the design and enhancement of license plate images based on kernel estimation using adaptive filter. Here the information patches are selected from the given images. From these images the edge prediction is performed. It means here it will determine the angle and length of the observations. After this kernel estimation operation is performed. Hence the proposed system can evaluate the images of real world and handle the motion of images when the license plate is unrecognizable. At last the proposed system gives effective output compared to other systems.
\end{abstract}

KEY WORDS: Kernel Estimation, Edge Prediction, Adaptive Filter, License plate.

\section{INTRODUCTION}

Basically, in the trouble making vehicles, license plate most important role and acts as an ID which very unique compared to others. In the high ways and the cities, the detection of over speed system is violated. But while moving the vehicle, at the time of exposure the image is blurred. There will be an impact on the time of exposure and this mainly depends on the situations which are illuminated [1]. For example when the time of exposure is $1 / 300$ second then the speed of vehicle is 60 miles per hour. The license plate of the displacement is about nine centimeters. For this the kernel length is estimated about pixels of 45 and the size is maintained by $140 \times 440$ pixels. There is an angle between the planes of horizontal and imaging plane. Here the license plate for a blur image is not neglected. In some other cases, the exposure time will be semantic in nature. While the vehicle is in motion, the blur image illuminates poor situations. Hence to get fully exposed image, the time of exposure should be prolonged. The blur image is obtained and susceptible in nature. High speed and high resolution image are obtained from the digital cameras and videographer.

Revised Manuscript Received on December 13, 2019.

* Correspondence Author

${ }^{1}$ B.HARICHANDANA, Research Scholar , Department of Computer Science and Technology, S.K.University, Anantapur, Andhra Pradesh

${ }^{2}$ K.LAVANYA, Research Scholar, Department of Computer Science and Technology, S.K.University, Anantapur, Andhra Pradesh.

${ }^{3}$ P.SUMALATHA, Assistant Professor, Sri Vani Institute of Management and Sciences, Anantapur, Andhra Pradesh.

${ }^{4}$ C.KRISHNAPRIYA, Assistant Professor, Department of Computer Science and I.T, Central University Of Andhra Pradesh, Anantapur, Andhra Pradesh

In most severe conditions, it means the effect of blurring is obtained in high form at the time of over speed motion of vehicle This will be difficult to recognize the plates from the detection. Hence in this the enhancement of kernel estimation is done by using license plate images [2]. The main intent of the proposed system is to recover the clue which is semantic in nature and which can be identified.

Here an image is captured when the vehicle is at high speed and the main intent is to capture the image which is blurred. In image processing applications, the blind image de-blurring/de-convolution techniques has attained very important place. In this real world various types of address is challenged. Hence the image blurring is mathematically represented as shown in below equation (1):

$\mathrm{B}(\mathrm{x}, \mathrm{y})=(\mathrm{k} * \mathrm{I})(\mathrm{x}, \mathrm{y})+\mathrm{G}(\mathrm{x}, \mathrm{y})$

Here the terms $\mathrm{B}, \mathrm{I}$ and $\mathrm{K}$ represent the image which is blurred. Here image is sharpening by recovering the image using kernel estimation [3-4].

The additive noise is represented by using the parameter $\mathrm{G}$ and the operator of convolution is represented as $*$. The image which is sharp is represented by term I. The relationship between the kernel and the blind image is unknown in this condition. Basically, the kernel estimation in this system is invariant in nature. The Blind image deblurring is generally classified into two types they are uniform and non uniform BID.

The point spread function is generally known as the uniform BID and in the same way the non uniform is known as the function of unspread. By using the uniform and non uniform methods, BOD gives effective results. It avoids the failure occurred in the system and in the same way discontinuities occurred in the system. Hence to get an exact solution the kernel estimation is done by using the de-blurred image now to this image BID process is applied to get effective output. The accurate results are obtained by estimating the two step strategy and non uniform BID approach. This method will restore the images in effective way.

But here the license plate algorithm gives effective output compared to BID. The license plate approach will overcome the problems that are occurred in the BID. The distinctive characteristics are obtained to improve the quality of visual. The results obtained from the approaches are recognizable. The license plate de-blurring technique will be categorized into three aspects [5]. 


\section{Design and Enhancement of License Plate Images Based on Kernel Estimation using Adaptive Filter}

To obtain a big scene after capturing of images, a surveillance camera is designed. From the captured image the license plate will occupy the small region. Hence this gives effective information which is required for the enhancement. The kernel estimation produces the information of edge which is available from the blurred images. Hence the large scale images will give robust and accurate output based on the estimation of kernel.

\section{RELATED WORK}

The main intent to get a blur image in digital imaging is poor quality. There are mainly two reasons to get a blur image, one is because of camera shake and another one is out of focus. By using the shift invariant kernel plus method the image blurring process is performed. It is represented as shown from below equation (2):

$$
\mathrm{f}=\mathrm{k} * \mathrm{~g}+\mathrm{n},
$$

The operator of discrete convolution is shown by using the parameter ' $*$ '. The image which is clear can be represented by using the parameter $\mathrm{g}$. the observation of blurry image is represented by using the parameter $f$. the kernel blur image is represented by using the parameter $\mathrm{k}$. At last the noise in image is represented by using the term $n$.

The effective solutions are obtained by introducing the models of spatially variant blurring. Hence the spatially variant and spatially invariant models are related to each other. This both models are represented by using matrix vector form and this is shown from below equation (3):

\section{$\mathrm{f}=\mathrm{Ag}+\mathrm{n}$}

The column wise vector forms are represented by using the parameters $\mathrm{g}, \mathrm{n}$ and $\mathrm{f}$. Here $\mathrm{A}$ is nothing but a matrix which is blurred in nature. Here smoothing process is obtained by using the spatially invariant blurring process. After applying this technique there is a shift in the position of bits spatially. Here the blurry image is estimated by using the de blurring process. Basically, two problems are occurred by estimating the process. They are given as 1) clear image $\mathrm{g}$ is estimated and 2 ) blur kernel image is estimated. These two problems are very complicated while estimating the process. Earlier, to avoid these problems, two methods are introduced. They are blind image de-blurring and non blind image de-blurring. Generally, the non blind image blurring process will obtain noise in the image and attenuates high frequency response. By attenuating the high frequency response the noise in image will double. To remove this noise and amplify the noise reverse operation is performed. Hence the noise is eliminated by introducing the reverse process. The blur kernel estimated image is free from errors and gives effective output. The kernel estimation of license plate images is implemented using hardware and software blind motion activities.

By using the different data sets the blur kernel estimation is performed. The blurring process is spatially varied in practical applications. Matrix A is introduced in blur motion to estimate the error presented in the system. Sensitivity is obtained in the blurring process to address the blur image. Accelerated Proximal Gradient (APG) is introduced to remove the additive image noise in the system. The boundary value problems which are occurred in the existed system are avoided by using proposed system estimation. The blur images are basically spatially variant in nature and gives effective result.

\section{LITERATURE SURVEY}

Marginalization and Maximum posterior methods are introduced from the interface of Bayesian. The recovery and estimation problem is reduced by using the parameters of specific kernel. Latent image is obtained by using the Maximum posterior methods. Levin introduced the concept of gradient sparsity prior which is corresponding to the kernel image and sharp image. This solution will avoid the problem of no blur solution.

The spatially random distribution concept is introduced by the Shan. This concept will smooth the images and reduce the noise in the images. This will improve the performance by enhancing the prediction of blur image. In this the edge prediction concept is critical to de-blur the performance. Text detection algorithm is introduced to enhance the blur image. But this introduced method is very complex in nature. It does not give effective background images prediction.

The power spectrum is calculated by estimating the kernel method. This concept is introduced by the Goldstein and Fatal. This is defined by the introducing the spectral whitening formula. There will be impressive results in the convex kernel regularize. This concept will resort the information of sharp images highly. The estimation of paired images is done by using blur kernel and this concept is introduced by the Zhang and Yuan. The de-blurring problems overcome by introducing the spatial de-blurring process and this is introduced by the Tai. This concept gives low resolution on the frame or video. In real time applications hardware observation is infeasible. Firstly, true solution is obtained by using gradient power and next size of kernel is estimated from the edges.

The method of marginalization is introduced by the Wang which improve the robustness of a blur image. But it gives small kernel solutions in terms of size. But it gives effective result in terms of speed and blurriness. From below section the estimation of license plate images based on kernel estimation model is discussed.

\section{LICENSE PLATE IMAGES BASED ON KERNEL ESTIMATION MODEL}

Basically, the blur image and the sharp image are modeled by using the following representation. The below equation (4) shows the blur image represented which is the combination of sharp and kernel images:

$$
y=f(x * k)+e n G
$$

The non linear function which is obtained from the output is represented by using the term $\mathrm{f}(\cdot)$, here (') is an operator. The non Gaussian distribution noise is represented by using the term enG. The range of image representation is done based on the saturation value records. Here the contrast of additive model for blur image is represented by using the below equation (5)

$\mathrm{y}=\mathrm{k} * \mathrm{x}+\mathrm{e}+\mathrm{o}$ Blue Eyes Intelligence Engineering \& Sciences Publication 
The Gaussian noise obtained in the image is represented by using the parameter e. The outliers in the blur image are represented by using the parameter $\mathrm{o}$. The term $\mathrm{f}(\mathrm{x}$ * $\mathrm{k})-\mathrm{x} * \mathrm{k}$ is obtained by absorbing the resource of outliers. Hence the both outlier and Gaussian noise are not related to each other. From below equation (6) shows the additive model of blur image representation:

$y=H(k) x+e+o=A(x) k+e+o$,

The convolution matrices is associated by using the parameters $\mathrm{x}$ and $\mathrm{k}$. but this is done based on the two conditions they are $\mathrm{A}(\mathrm{x}) \in \mathrm{R} \mathrm{n} \times \mathrm{m}$ and $\mathrm{H}(\mathrm{k}) \in \mathrm{R} \mathrm{n} \times \mathrm{n}$. Now based on these conditions the Gaussian noise (e) is handled easily in the kernel estimation process. While distributing the information there will be no prior knowledge. Hence by using kernel estimation small amount of portion of pixels is estimated and contaminated in reasonable way.

Here a binary vector form is introduced by using the limit $v \in$ $\{0,1\} \mathrm{n}$. Here the pixel values are indexed based on the kernel estimation and outlier. In case of 0 , the kernel estimation will be excluded based on outlier and in case of 1 , the kernel estimation is estimated by using inliers. The below figure (1) shows the architecture of license plate images based on kernel estimation model.

Figure (1) consists of mainly informative patches, edge prediction technique, kernel estimation based on noise, adaptive filter and kernel refinement. Here first $\mathrm{N}$ input images are given from the blurry image series. The patches of images are selected in a particular form. The edge prediction will determine the size of the images. Now to avoid the noise occurred in the images kernel estimation is performed. The obtained output image is filtered using the adaptive filter technique. This will remove the unnecessary noise occurred in the images. The kernel refinement will take the blur images and gives clarity of that license plate image number in effective way. Hence the proposed system gives effective output.

IMAGES BASED ON KERNEL ESTIMATION MODEL The kernel estimation for multi frames is discussed here. Here the series format is used based on the multi frames. Now these images are spatially invariant in nature. The patches of images in the kernel estimation are determined from the final course of kernel concept. Hence the below equation (7) shows the single image course kernel:

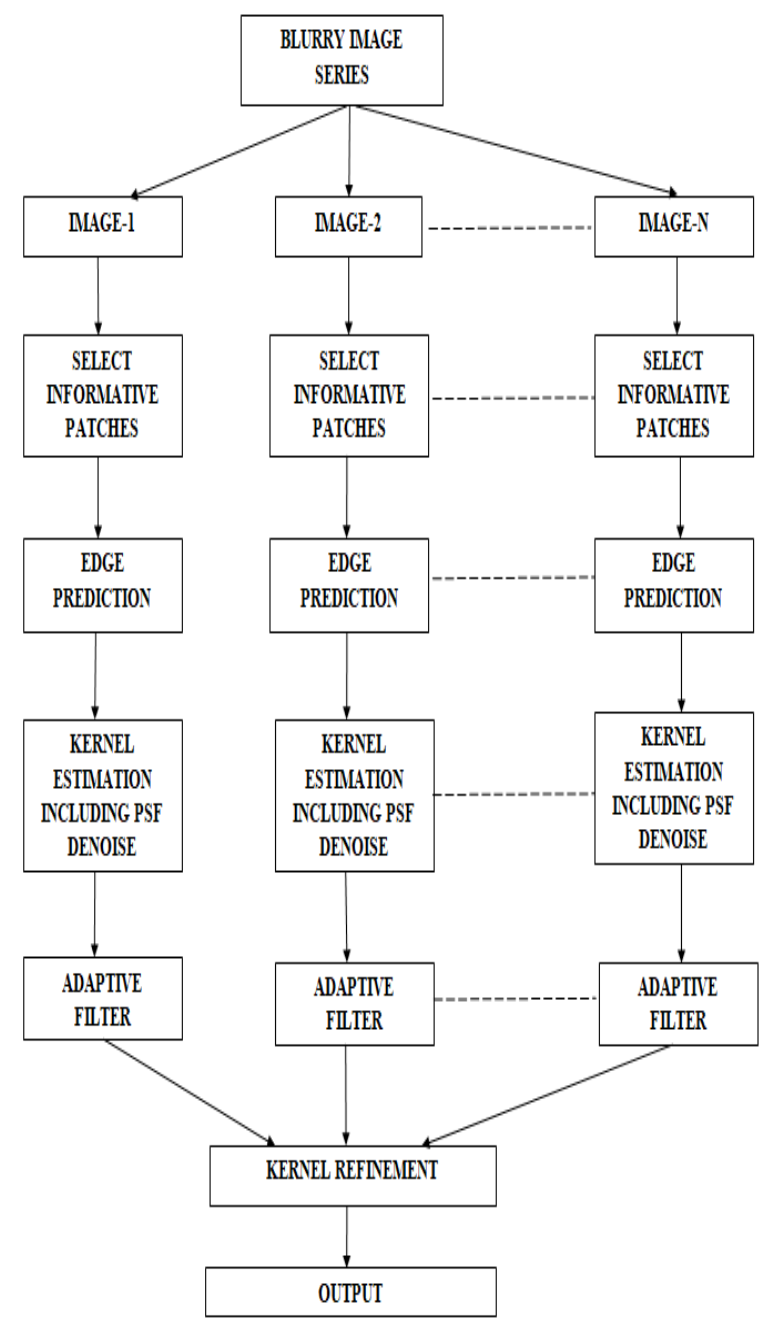

Fig. 1: ARCHITECTURE OF LICENSE PLATE

$$
h=\sum_{i=1}^{m} \frac{\delta(i) \times h_{\text {coarse }}(i)}{\sum_{j=1}^{m} \delta(j)}
$$

Here ' $\mathrm{i}$ ' is nothing but a patch which is estimated from the edge factor. The series of blurry images are represented by using ' $m$ ' parameter. Now this kernel image is transformed using different techniques and as is represented in below equation (8).

$$
h_{\text {series }}(i)=\mathrm{T}_{i}\left(h_{\text {real }}\right)+n_{i}
$$

Hence from this it can observe that the proposed system gives effective output compared to others in terms of speed and robustness.

\section{RESULTS}

The below figure (2) shows the enhancement of license plate images using kernel estimation. 


\section{Design and Enhancement of License Plate Images Based on Kernel Estimation using Adaptive Filter}

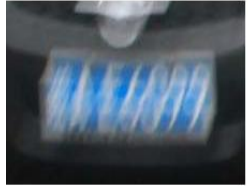

(a)

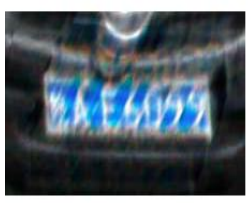

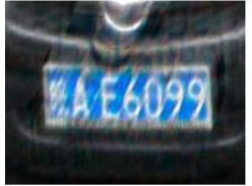

(b)

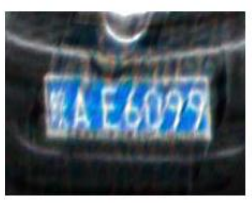

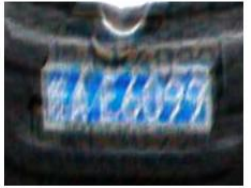

(c)

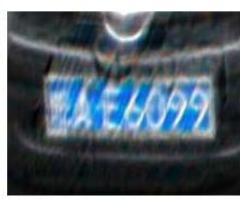

Fig. 2: (a) BLURRED IMAGE. (b) $\left(\theta=66.67^{\circ}, \mathrm{l}=\mathbf{2 9 . 8 7}\right)$. (c) $\left(\theta=66.67^{\circ}, I=24.87\right)$. (d) $\left(\theta=66.67^{\circ}, I=34.87\right)$. (e) $(\theta=$ 61.67, $\mathrm{I}=29.87)$. (f) $\left(\theta=71.67^{\circ}, \mathrm{l}=29.87\right)$.
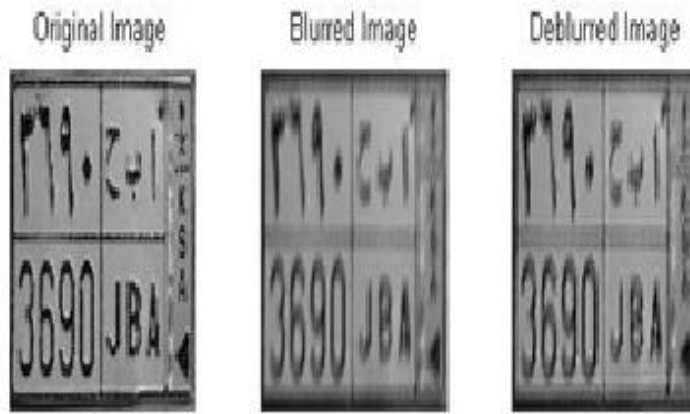

Fig. 3: REPRESENTATION OF KERNEL ESTIMATION

\section{CONCLUSION}

By overcoming the computations of previous method, in this paper, the design and enhancement of license plate images using kernel estimation using adaptive filter architecture is implemented. This system will restore the high quality images because of kernel refinement approach. The selection patch technique will reduce the cost of computation. The simulation result shows that there is best visual quality and this can handle the multiple images by shifting the positions.

\section{REFERENCES}

1. R. Wang, D. Tao, "Recent Progress in Image Deblurring," Computer Vision and Pattern Recognition (CVPR), 2014 IEEE Conference on, 2014.

2. D. Perrone, P. Favaro, "Total Variation Blind Deconvolution: The Devil is in the Details," Computer Vision and Pattern Recognition (CVPR), 2014 IEEE Conference on, 2909-2916 (2014).

3. X. Li, Z. Shicheng and J. Jiaya, "Unnatural LO Sparse Representation for Natural Image Deblurring," Computer Vision and Pattern Recognition (CVPR), 2013 IEEE Conference on, 1107-1114 (2013).

4. X. Zhu, P. Milanfar, "Removing Atmospheric Turbulence via Space-Invariant Deconvolution," Pattern Analysis and Machine Intelligence, IEEE Transactions on, 35(1), 157-170 (2013).

5. J. Hui and W. Kang, "A two-stage approach to blind spatially-varying motion deblurring," Computer Vision and Pattern Recognition (CVPR), 2012 IEEE Conference on, 73-80 (2012).

6. X. Li and J. Jiaya, "Depth-aware motion deblurring," Computational Photography (ICCP), 2012 IEEE International Conference on, 1-8 (2012).

7. L. Xu, Q. Yan, Y. Xia and J. Jia, "Structure extraction from texture via relative total variation," ACM Transactions on Graphics (TOG), 31(6), 1-10 (2012).

8. X. Zhu, F. Šroubek and P. Milanfar, "Deconvolving PSFs for a Better Motion Deblurring Using Multiple Images," Computer Vision ECCV, 636-647 (2012).

9. A. Levin, Y. Weiss, F. Durand and W. T. Freeman, "Understanding Blind Deconvolution Algorithms," Pattern Analysis and Machine

Intelligence, IEEE Transactions on, 33(12), 2354-2367 (2011) [10] R F. Dilip Krishnan, "Fast Image Deconvolution using Hyper-Laplacian Priors," Advances in Neural Information Processing Systems (NIPS), 1033-1041(2009)

\section{AUTHOR PROFILE}
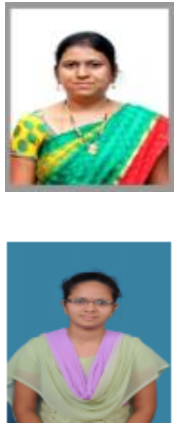

K.LAVANYA completed M.sc and pursuing Ph.D from Department of Computer Science and Technology, S.K.University, Anantapur.

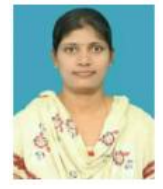

Dr.P.SUMALATHA completed M.Sc and Ph.D from Sri Krishnadevaraya University, Anantapur. At present she is working as Assistant Professor, Sri Vani Institute Of Management And Sciences Anantapur, Andhra Pradesh.

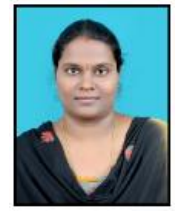

C.KRISHNA PRIYA completed M.C.A and Ph.D from Sri Krishnadevaraya University, Anantapur. At present she is working as Assistant Professor, Department Of Computer Science And I.T, Central University of Andhra Pradesh, Anantapur, Andhra Pradesh. 